\title{
Histological Analysis of Collagen Fibers in Patients with Diabetes Mellitus and Periodontal Disease
}

\section{Popescu MR*, Surlin P, Rauten AM, Dragomir L and Olteanu M}

Department of Prosthetics and Occlusology, Faculty of Dental Medicine, University of Medicine and Pharmacy of Craiova, Romania

*Corresponding author: Mihai Raul Popescu, Department of Prosthetics and Occlusology, Faculty of Dental Medicine, University of Medicine and Pharmacy of Craiova, 2 Petru Rareş Street, 200349 Craiova, Romania; Tel: +40722-557 407; E-mail: popescumihairaul@yahoo.com

Rec date: Apr 03, 2014, Acc date: June 14, 2014, Pub date: June 16, 2014

Copyright: (c) 2014 Popescu MR, et al. This is an open-access article distributed under the terms of the Creative Commons Attribution License, which permits unrestricted use, distribution, and reproduction in any medium, provided the original author and source are credited.

\begin{abstract}
The researchers suggest that diabetes increases the degradation of the newly synthesized collagen from the conjunctive tissue. The altered metabolism of the collagen may predispose the diabetic patients not only to periodontal disease, but also to some other anomalies such as defective healing. The purpose of the study was to go through a histological analysis of the collagen fibers from the chorion in patients with diabetes mellitus and periodontal disease.
\end{abstract}

Materials and methods: In our study we examined a total of 68 patients diagnosed with diabetes mellitus type 1 and 2. In these patients we achieved simple gingivectomy or tooth extraction in order to obtain gingival tissue fragments. The preparations included at paraffin and then stained with Hematoxylin-Eosin and trichromic after Goldner-Szeckeli method.

Results: In the patients with diabetes mellitus with an evolution of less than 10 years the collagen fibers appeared as dissociated by the inflammatory cells that were inserted among them. It was also noted the fragmentation of collagen fibers and sometimes even their lysis with subepithelial connective tissue disorganization. In patients with diabetes for over 10 years there has been noticed a plentiful collagen sclerosis, zones of collagen hyalinization with an increased number of fibrocytes.

Conclusion: The extracellular matrix is altered in the investigated patients with destructive lesions of collagen or collagen sclerosis.

Keywords: Diabetes mellitus; Periodontitis; Collagen fibers

\section{Introduction}

The researchers concentrated their efforts on different aspects of the host response in patients with diabetes mellitus, including the metabolism of the collagen [1]. Some findings suggest that diabetes increases the degradation of the newly synthesized collagen from the conjunctive tissue [2].

The altered metabolism of the collagen may predispose the diabetic patients not only to periodontal disease, but also to some other anomalies such as defective healing [3-5]. There has been noticed that patients with diabetes mellitus exhibit an intense activity of the collagenases [6,7] and a decrease of the fibroblastic collagen synthesis in the gingival tissue $[7,8]$. The neutrophils represent one of the cellular sources for the intense collagenase activity. The fibroblasts however may contribute to the collagen excess $[9,10]$.

Assuming the existence of a relationship between diabetes and the periodontal disease, we emphasize the importance of the interdisciplinary collaboration to counteract the local and systemic effects of periodontal disease.

The purpose of the study was to go through a histological analysis of the collagen fibers from the chorion in patients with diabetes mellitus and periodontal disease.

\section{Material and Methods}

The investigated patients were selected from a total number of 117 persons diagnosed with diabetes mellitus (DM) in The Nutrition and Metabolic Diseases Clinic of The Clinical Emergency County Hospital from Craiova and from Periodontal Department of Faculty of Dentistry from Craiova. A descriptive observational study was conducted over a period of two years, between 2006 and 2008.

The including criteria referred to those specific symptoms for periodontal disease as gingival inflammation, bleeding and gingival recession with deep pockets or, on the contrary, gingival overgrowth, mobility or loss of dental units. We excluded patients with medical history of systemic diseases or drugs treatments known to have hypertrophy effect over gingival mucosa (hydantoin, calcium antagonists and cyclosporine).

We selected a number of 68 patients from the investigated persons with DM diagnosis who accepted dental treatment and presented suggestive alterations for periodontal disease as follows: 12 patients with DM type 1 and 56 patients with DM type 2. The glycated hemoglobin (HbAlC) was also tested and its values indicated a poor metabolic control: HbA1C 6-10\% for 11 patients with type $1 \mathrm{DM}$ and 37 patients with type $2 \mathrm{DM}$; HbA1C $>10 \%$ for 2 patients with type 1 $\mathrm{DM}$ and 13 patients with type $2 \mathrm{DM}$. Only 5 patients with type $2 \mathrm{DM}$ had good metabolic control (HbA1C 4-6\%). 
Citation: Popescu MR*, Surlin P, Rauten AM, Dragomir L, Olteanu M (2014) Histological Analysis of Collagen Fibers in Patients with Diabetes Mellitus and Periodontal Disease. J Cytol Histol S4: 008. doi:10.4172/2157-7099.S4-008

Page 2 of 4

The patients were divided into 2 groups referred by the DM evolution period. 35 patients had a DM evolution for over 10 years (2 patients with type $1 \mathrm{DM}$ and 33 type 2 ) and 33 patients with a DM evolution of less than 10 years (10 patients with type $1 \mathrm{DM}$ and 23 type 2).

These patients were carried simple gingivectomy or tooth extraction with gingivectomy. We performed simple gingivectomy for all the investigated cases and also tooth extraction at patients with dental mobility. The extractions were made for all compromised dental units and regarding gingivectomy, it was performed in different areas, both areas with advanced, moderate or small hypertrophy, and areas with no clinical evident hypertrophy typical for patients of more than 65 years old and type $2 \mathrm{DM}$.

The study was approved by the Ethic Committee of University of Medicine and Pharmacy from Craiova through agreement no. 20 for harvesting gingival fragments from patients with periodontitis.

We performed histopathological examination of the gingival tissue fragments. For this purpose the obtained mucosal fragments were fixed in neutral $10 \%$ formalin solution for 48 hours and then included in paraffin by the usual technique.

The mucosal fragments were processed by the usual technique of inclusion into paraffin. The preparations were then stained with Hematoxylin-Eosin and trichromic after Goldner-Szeckeli method.

\section{Results and Discussion}

Considering DM period of evolution, we discovered that many of the investigated patients with the most severe periodontitis have been suffering from DM for over 10 years. But the periodontal alterations of these patients were registered along with a poor glycemic control $(\mathrm{HbAlC}>10 \%)$ and a bad hygiene. The same frame was met in patients who suffered from DM of less than 5 years and however presented advanced forms of periodontal disease. Therefore we cannot state that the evolution period of DM represents a cause for severe periodontal structures alterations for the patients in our study, but we can submit the hypothesis that periodontal disease evolved to severe destructive forms in the presence of a poor hygiene, a poor metabolic control and probably influenced by the evolution period of DM.

The histological study of the extra cellular matrix mainly focused on the analysis of changes in the collagen fibers. The trichromic GoldnerSzeckeli staining was really useful to highlight these changes.

In the patients with diabetes mellitus with an evolution of less than 10 years the collagen fibers appeared as dissociated by the inflammatory cells that were inserted among them (Figure 1). The interstitial oedema and the hemorrhagic infiltrates were present in most of the cases and they also contributed to disorganization of the chorion architecture. It was also noted the fragmentation of collagen fibers and sometimes even their lysis with subepithelial connective tissue disorder. On the trichromic GS stained preparations there were present only rare collagen fibers (dissociated by the immune system cells), especially in the superficial chorion (Figure 2), and also in the deep chorion. A good part of the missing collagen fibers were replaced by inflammatory cells. Only isolated collagen fibers and rare fibroblasts were found on the histological preparations.

A plentiful collagen sclerosis, zones of collagen hyalinization (with predominant perivascular localization) with an increased number of fibrocytes were noticed for patients with a long evolution of the periodontal disease along with the reducing of the inflammatory intensity at chorion level (Figure 3). The collagen fibers had a thick bands positioning with continuous aspect, which did not comply with the original architecture. They have also totally occupied the lamina propria (Figure 4). In some places collagen bands had a discontinuous aspect and they were fragmented.

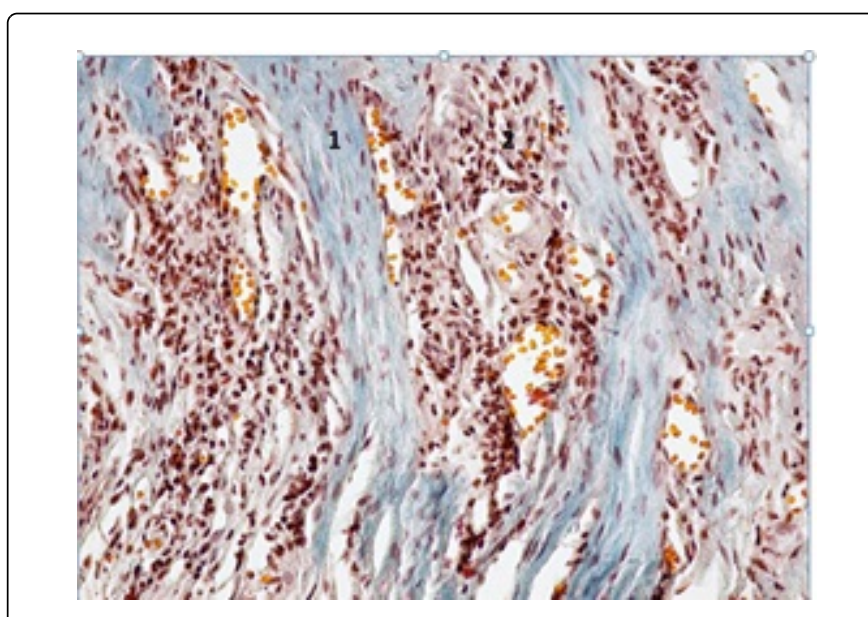

Figure 1: Collagen dissociation (1) by inflammatory infiltrate (2) (trichromic GS stain, ob. 200x)

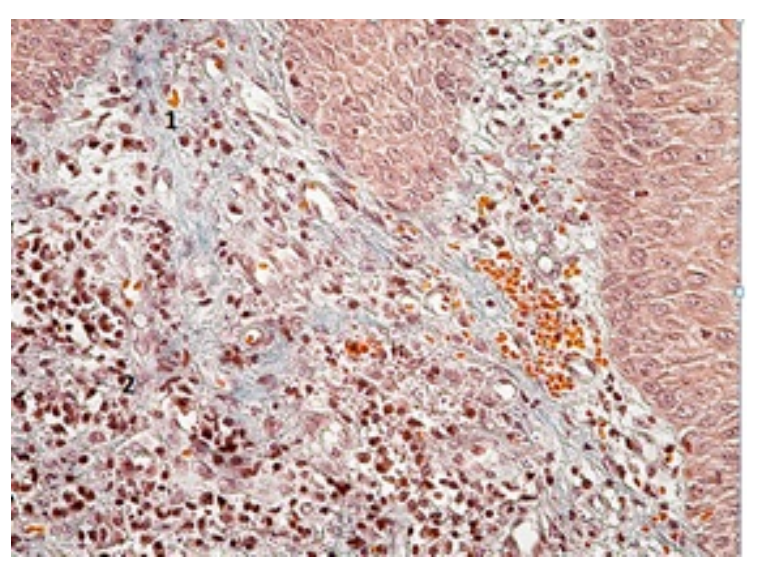

Figure 2: Rare collagen fibers in superficial chorion (1), included into the mass of the immune system cells (2) (trichromic GS stain, ob. 200x)

The process of interstitial collagen sclerosis was associated with the superjacent epithelium atrophy, with the deletion of the interpapillar ridges and tendency to rectilinear epithelium-chorion limit. We also observed vascular sclerosis accompanied by vascular lumen reduction.

We noticed in our study that for DM patients with an evolution of less than 10 years the collagen fibers appeared dissociated by the inflammatory cells that inserted among them. The interstitial oedema and interstitial hemorrhagic infiltrates encountered in many cases contributed to the chorion architecture destruction. We also noticed the collagen fibers fragmentation and sometimes their lysis with the destruction of the subepithelial conjunctive tissue. Only rare collagen fibers were present on trichromic GS staining preparates, most in 
superficial and also in profound gingival chorion. Most of the missing collagen fibers have been replaced by the inflammatory cells, on the histological preparates been present only isolated collagen fibers and rare fibroblasts. Monea et al. found that the degradation of connective tissue is present in diabetic subjects, due to a rich inflammatory infiltrate, destruction of reticular fibers and accumulation of dense collagen fibers (fibrosis) [11].

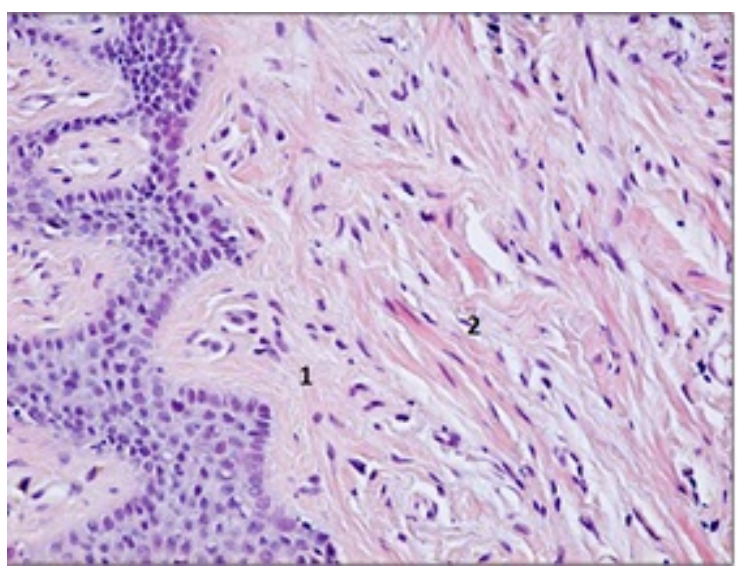

Figure 3: Collagen sclerosis in superficial (1) and deep chorion (2) (HE stain, ob. 200x)

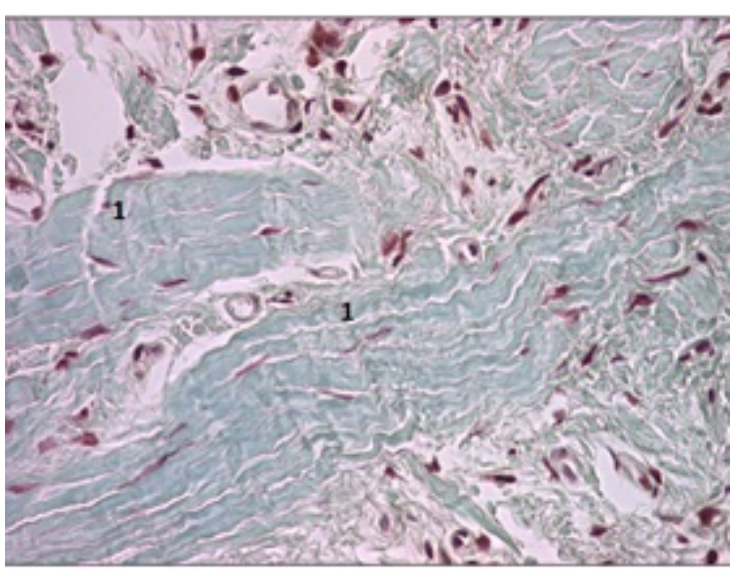

Figure 4: Collagen sclerosis in thick bands (1) into the chorion (trichromic GS stain, ob. 200x)

In diabetics the periodontium is probably affected by the increase in collagenase activity and by the functional anomalies of PMN degranulation as a source of collagenase from the crevicular fluid, also by other metabolic anomalies of the fibroblasts from periodontal ligament [12]. However the collagen metabolism is altered in diabetic patients. Hyperglycemia is associated with the increase of collagenase and protease activity at gingival level, as demonstrated by studies on rats [13].

An abundant collagen sclerosis, mostly perivascular hyalinizations, an increased number of fibrocytes, low intensity inflammatory infiltrates and collagen fibers dissociated by the infiltrate in the chorion have been present in patients with old periodontal disease and uncontrolled DM of more than 10 years evolution. This time the collagen fibers were arranged in thick bands that did not respect the initial architecture and occupied the whole lamina propria. The interstitial collagen sclerosis with thick collagen bands forming have been often associated with overlying epithelium atrophy, interpapillary ridges removal, straightening of epithelium-chorion limit and also vascular sclerosis. Patients with a DM history of 5 to 15 years and/or mostly atrophic alterations presents subepithelial capillary sclerosis and an important stromal sclerosis [14].

The collagen metabolism in patients with DM is corrupted and it is one of the components that causes a delayed healing. In order to study the details of the delayed healing, there has been made a series of studies on animals and there has been found that hyperglycemia is associated with an increase of collagenase and protease activity in rats [13].

The altered metabolism of the collagen and AGE (advanced glycation end products), with altered PMN function (on chemoattraction, adhesion and phagocytosis) which may lead to a deficient host response to infection represent factors that contribute to the development of the periodontal disease in the diabetic patients [15]. It seems that the synthesis, the maturation and the homeostasis of collagen are affected by the glucose level. In the diabetics there is a small increase in proliferation of fibroblasts, as well as a reduced synthesis of collagen and glycosaminoglycans, also an increase in the collagenase activity. AGE formation plays an important role in the complications of diabetes mellitus. AGE accumulates in the chronic hyperglycemia and impaires the function of various components of the extra cellular matrix, but also modifies the matrix-matrix and matrixcells interaction. This alteration has an adverse effect over the target tissues, especially regarding the collagen stability and the vascular integrity. AGE formation causes the development of crossed links between the collagen molecules, resulting into the decrease of the solubility and the turnover rate [15].

The susceptibility to infections in the diabetic patients is given by the defective PMN function, the insulin resistance and the vascular changes. In addition, the patients with diabetes have a low capacity for recovery characterized by the decrease in the amount of collagen, associated with its poor contractile strength. The decrease in collagen synthesis by fibroblasts and the increase of collagenase production plays an important role in the reduced recovery.

Glycation of the collagen fibers from the edges of the wound leads to a reduction of its solubility and to the cancellation of the remodeling. The increased levels of collagenase degrade the newly synthesized collagen fibers. Also the monocytes phenotype transformation from a regenerative-repairing into an inflammatory one may be responsible for alternation of the wound recovery and for an exaggerated inflammatory response in the diabetic patients $[15,16]$.

\section{Conclusions}

The extracellular matrix of the gingival chorion is altered in the investigated patients. Patients with DM under 10 years evolution presented frequent destructive lesions of the collagen. Instead patients with DM for over 10 years evolution presented collagen sclerosis that does not respect the original architecture of the tissues. 
Citation: $\quad$ Popescu MR*, Surlin P, Rauten AM, Dragomir L, Olteanu M (2014) Histological Analysis of Collagen Fibers in Patients with Diabetes Mellitus and Periodontal Disease. J Cytol Histol S4: 008. doi:10.4172/2157-7099.S4-008

Page 4 of 4

\section{References}

1. Ejeil Al, Gaultier F, Igondjo-Tchen S, Senni K, Pellat B, et al. (2003) Are cytokines linked to collagen breakdown during periodontal disease progression? J Periodontol. 74: 196-201.

2. Narayanan AS, Clagett JA, Page RC (1985) Effect of inflammation on the distribution of collagen types I, III, IV and V and type I trimer and fribonectin in human gingivae. J Dent Res 64: 1111-1116.

3. Narayanan AS, Page RC (1983) Connective tissues of the periodontium: a summary of current work. Coll Relat Res 3: 33-64.

4. Seppälä B, Sorsa T, Ainamo J (1997) Morphometric analysis of cellular and vascular changes in gingival connective tissue in long-term insulindependent diabetes. J Periodontol 68: 1237-1245.

5. Martinez EF, Machado de Souza SO, Corrêa L, Cavalcanti de Araújo V (2000) Immunohistochemical localization of tenascin, fibronectin, and type III collagen in human dental pulp. J Endod 26: 708-711.

6. Kaplan R, Mulvihil J, Ramamurthy N, Golub L (1982) Gingival collagen metabolism in human diabetics. J Dent Res. 61: 275.

7. Ryan ME, Carnu O, Kamer A (2003) The influence of diabetes on the periodontal tissues. J Am Dent Assoc 134 Spec No: 34S-40S.

8. el-Kishky M, Mahfouz SA, el-Habbak SM (1986) An in vitro study of hydroxyproline synthesis by gingival fibroblasts in patients with juvenile diabetes. Egypt Dent J 32: 15-27.

9. Liu R, Desta T, He H, Graves DT (2004) Diabetes alters the response to bacteria by enhancing fibroblast apoptosis. Endocrinology 145: 2997-3003.
10. Marigo L, Cerreto R, Giuliani M, Somma F, Lajolo C, et al. (2011) Diabetes mellitus: biochemical, histological and microbiological aspects in periodontal disease. Eur Rev Med Pharmacol Sci 15: 751-758.

11. Monea A, Mezei T, Monea M (2012) The influence of diabetes mellitus on periodontal tissues: a histological study. Rom J Morphol Embryol 53: 491-495.

12. Giju GB, Padiyath S (2010) Gingival vascular basement membrane changes in diabetes mellitus. Oral and Maxillofacial Pathology Journal, 1: 25-28.

13. Grant-Theule DA (1996) Periodontal disease, diabetes and immune response: a review of current concepts. J West Soc Periodontol Periodontal 44: 69-77.

14. Balakhonov LV, Nepomnyashchikh LM, Aidagulova SV, Bakarev MA, Vlasova LF. (2006) Structural reactions of the buccal mucosa in diabetic parodontopathy. Bull Exp Biol Med 142: 633-636.

15. Balasundaram A, Ponnaizan D, Parthasarathz H (2010) Diabetes mellitus - A periodontal perspective. SRM University Journal of Dental Sciences 1: 79-85.

16. Nalmas S, Mehta DS (1999) Diabetes mellitus - A systemic modifier in periodontal disease. Journal of Indian Society of Periodontology 2: 48-52. 